\title{
APLICACIÓN DE HERRAMIENTAS DE ADMINISTRACIÓN TERRITORIAL EN LA GESTIÓN DE RIESGOS NATURALES. CASO, VALLE DE LOS CHILLOS - ECUADOR.
}

\author{
APPLICATION OF LAND ADMINISTRATION TOOLS IN NATURAL RISK \\ MANAGEMENT. LOS CHILLOS VALLEY - ECUADOR, CASE.
}

\section{Dennis Paúl Ushiña Huera}

Carrera de Ingeniería Geográfica y del Medio Ambiente, Departamento de Ciencias de la Tierra yla Construcción, Universidad de las Fuerzas Armadas ESPE. Av. Gral Rumiñahui s/n Sangolquí, Ecuador. dpushina@hotmail.com

\begin{abstract}
RESUMEN
$\mathrm{El}$ aumento en la frecuencia y magnitud de desastres naturales alrededor del mundo debido a factores antrópicos ha ocasionado grandes pérdidas económicas y afectaciones a la salud y seguridad. Uno de los tratamientos más efectivos para disminuir los riesgos asociados a estos eventos es la prevención mediante una adecuada planificación, donde la administración territorial es capaz de proveer información y herramientas como infraestructuras de datos, marcos legales y políticas. El presente trabajo aplicó dentro del proceso de gestión del riesgo, las herramientas proporcionadas por la administración de tierras, mediante el diseño de una metodología para la gestión de riesgos, su implementación en parte de las posibles áreas afectadas por un eventual lahar del volcán Cotopaxi en el valle de Los Chillos y posterior generación de una primera versión del Sistema Catastral de Riesgos (SCR). La metodología propuesta permite identificar, evaluar, valorar y mitigar el riesgo, para su elaboración se tomó como base el proceso de gestión de riesgos dado en la AS/NZS ISO 31000:2009, la información disponible en los catastros inmobiliarios municipales, además, de un análisis de la realidad local. De su implementación se obtuvo una completa valoración del riesgo a nivel predial para el área de estudio repartida entre los cantones Quito y Rumiñahui; y una serie de recomendaciones enmarcadas en la planificación territorial para tratarlo. Los datos obtenidos fueron ingresados al SCR cuyo diseño siguió el modelo LADM (Land Administration Domain Model) descrito en la ISO 19152:2009 y fue puesto en marcha con ayuda del software STDM (Social Tenure Domain Model). La aplicación de herramientas de administración territorial en la gestión de riesgos naturales, en este caso, de la norma ISO 19152 LADM, los datos e infraestructuras catastrales, y la capacidad de elaborar políticas de ordenamiento territorial y uso del suelo, facilita y mejora la gestión de riesgos y toma de decisiones.
\end{abstract}

Palabras clave: gestión de riesgos, administración territorial, catastro, desastre natural. 


\begin{abstract}
The increase in the frequency and magnitude of natural disasters around the world due to anthropic factors has caused great economic losses and impacts on health and safety. One of the most effective treatments to reduce the risks associated with these events is prevention through proper planning, where the land administration is able to provide information and tools such as data infrastructure, legal frameworks and policies. The present work applied within the risk management process, the tools provided by the land administration, through the design of a methodology for risk management, its implementation in part of the possible affected areas by an eventual lahar of the Cotopaxi volcano in Los Chillos Valley and subsequent generation of the Cadastral Risk System (SCR) first version. The proposed methodology allows identify, evaluate, assess and mitigate the risk, for its elaboration the risk management process given in AS / NZS ISO 31000: 2009 was taken as a basis, as well as the information available in municipal real estate cadastres and an analysis of the local reality. From its implementation a complete risk assessment was obtained at the property level for the study area distributed between the Quito and Rumiñahui cantons; and a series of recommendations framed in territorial planning to deal with it. The data obtained were entered into the SCR whose design followed the LADM (Land Administration Domain Model) model described in ISO 19152: 2009 and was launched with the STDM software. The application of territorial administration tools in the management of natural hazards, in this case, of the ISO 19152 LADM standard, cadastral data and infrastructure, and the ability to develop land management and land use policies, facilitates and improves management of risks and decision making.
\end{abstract}

Keywords: risk management, land administration, cadastre, natural disaster.

\title{
INTRODUCCIÓN
}

El mundo ha evidenciado un aumento en la frecuencia y gravedad de desastres naturales tales como: terremotos, inundaciones, sequías, huracanes, derrumbes y tsunamis, que se han visto agravados por la intensificación de las actividades agrícolas, el crecimiento de la población, la presión sobre los recursos naturales y el cambio climático. Estos factores ocasionan un sin número de pérdidas por los efectos devastadores que provocan sobre el territorio y la propiedad tanto pública como privada; solamente entre el 2000 y 2005 , en promedio hubo 240 millones de personas afectadas, 80,000 vidas perdidas y $\$ 80$ mil millones en daños cada año (Baas, et al., 2008). Acorde a la base de datos EM-DAT (Emergency Events Database) del Centro de Investigación sobre Epidemiología de los Desastres (CRED) el número de muertos por desastres ha aumentado de forma constante desde el inicio de los registros de la base de datos en el año 1900 (IPCC, 2014; Mitchell, 2011). Al incremento en el número de desastres naturales se suma la acumulación de deficiencias históricas en la planificación y desarrollo de los territorios vulnerables, lo que intensifica sus efectos negativos debido al inadecuado uso del suelo y ocupación, como reflejo del modelo de desarrollo que ha prevalecido en un país o región (Correa, et al., 2011). Las deficiencias en la planificación territorial se deben en gran parte al escaso conocimiento técnico de los peligros naturales a los que está expuesto el territorio, una débil gestión de riesgos y deficiencias en los sistemas de administración territorial que dificultan entre otras cosas identificar la tenencia legítima de las tierras, especialmente en países en vías de desarrollo; impidiendo a las personas que poseen dichos derechos de propiedad y a los organismos competentes, llevar a cabo acciones de 
prevención y mitigación ante desastres (Mitchell, et al., 2014; Mitchell, 2011). Es así como se deja entre ver la estrecha relación existente entre la gestión de riesgos y la administración territorial. Un caso de estudio, como el realizado durante la recuperación y reconstrucción tras el terremoto de magnitud 7.6 en la escala Richter ocurrido en Nepal, demostró la importancia de un adecuado manejo del sistema de administración territorial antes y después de un desastre, ya que al mapear las relaciones entre las personas y la propiedad, es posible identificar a quienes deben y pueden recibir ayudas para la reconstrucción, facilitando la toma de decisiones (Unger, et al., 2019). Con la finalidad de tratar los riesgo desde el ambito territorial se han diseñado modelos basados en el estándar LADM que permiten gestionar las relaciones entre las personas y la propiedad incluyendo aquellas derivadas de un contexto de desastre natural (Unger, et al., 2019). Trabajos como "Innovative land use planning for natural Hazard risk reduction: A consequence-driven approach from New Zealand" de Saunders y Kilvington (2016), y "Using land administration for land risk management" de Potts (2013) no solo aplican las herramientas de administración territorial como ayuda para llevar los registros en la etapa pos desastre, sino, que incorporan al riesgo como factor fundamental dentro de la planificación territorial, en una de las primeras fases para el tratamiento del riesgo que sería la prevención, lo cual permite mejorar la toma de decisiones y generar e implementar prácticas y estrategias efectivas para alcanzar el desarrollo de comunidades resilientes.

En el contexto latinoamericano debido a la vulnerabilidad de la región frente a la ocurrencia de desastres naturales, organismos como la Comunidad Andina de Naciones (CAN) o Cooperación Alemana (GiZ) han dado elementos básicos sobre amenaza, vulnerabilidad y riesgo y su relación con el territorio en busca de facilitar la articulación del ordenamiento territorial y la gestión de riesgos de desastres (Vargas y Ramírez, 2009; Rojas, 2013). Sin embargo, pese a iniciativas puntuales a nivel regional y local, aún no se cuentan con verdaderos procedimientos y metodologías que permitan alcanzar una planificación territorial capaz de afrontar los riesgos asociados a los peligros naturales, aun cuando los problemas referentes a riesgos naturales pueden ser identificados con cierta facilidad y existen medidas de mitigación cuyos beneficios al reducir la vulnerabilidad son ampliamente mayores frente a los costos de no hacerlo. En América Latina y el Caribe, 90\% de los fondos internacionales destinados al manejo de amenazas naturales son usados en actividades de preparación, auxilio, rehabilitación y reconstrucción mientras solo un $10 \%$ se destina a la prevención, pese a conocerse que el método más efectivo para reducir el impacto negativo de estos eventos naturales es incorporar la evaluación y mitigación de amenazas naturales dentro de los procesos de planificación y desarrollo territorial (Mitchell, et al., 2014; OEA, 1991; UNISDR AM, 2016). El uso de información acerca del manejo de tierras y de las herramientas de administración territorial en la gestión de riesgos mejora las operaciones de emergencia y permite a los 'stakeholders' identificar, analizar, evaluar y seleccionar los tratamientos más apropiados para el riesgo, sin embargo, continúan limitados a sus usos históricos como es el cobro de impuestos (Potts, 2013).

Los sistemas de administración territorial son infraestructuras compuestas por diversas instituciones, marcos legales, información territorial y componentes técnicos que posibilitan la implementación de políticas y estrategias territoriales, y poseen el gran potencial de facilitar la gestión de múltiples riesgos. El desarrollo del presente proyecto tuvo por objetivo aplicar las herramientas e información que es capaz de proporcionar la administración territorial dentro de la gestión de riesgos naturales, con esta finalidad se diseñó y adaptó una metodología para la gestión de riesgos desde el ámbito territorial, consecuentemente fue implementada en parte de las posibles áreas afectadas por un eventual lahar del volcán Cotopaxi en el valle de Los Chillos y posteriormente fue generada una primera versión del Sistema Catastral de Riesgos (SCR). La metodología propuesta toma como base el proceso de gestión del riesgo dado en la AS/NZS 
ISO 31000:2019 desarrollada para Nueva Zelanda y Australia; la información disponible en los catastros inmobiliarios municipales; y un análisis de la realidad local, para proponer una metodología aplicable en el Ecuador, la cual utiliza los insumos proporcionados por la administración de tierras para identificar, evaluar, valorar y mitigar el riesgo. De su implementación se obtuvo una completa valoración del riesgo a nivel predial para el área de estudio repartida entre el Distrito Metropolitano de Quito (DMQ) y el Gobierno Autónomo Descentralizado de Rumiñahui (GADMUR); y una serie de recomendaciones enmarcadas en la planificación territorial para tratar el riesgo. A partir de estos resultados se generó el SCR basado en el modelo LADM (Land Administration Domain Model) descrito en la ISO 19152:2009 y puesto en marcha con ayuda del software STDM. En el modelo LADM se consideran los derechos, restricciones y responsabilidades para cada uno de los interesados (personas u organizaciones) en cada una de las unidades espaciales de acuerdo al nivel territorial; este caso en particular, estuvo enfocado hacia el nivel predial.

\section{METODOLOGÍA}

\section{DISEÑO DE LA METODOLOGÍA}

Como punto de partida se realizó el análisis del sistema de administración territorial y del manejo actual de los catastros inmobiliarios del GAD Municipal de Rumiñahui, el GAD Metropolitano de Quito y la norma técnica para catastro inmobiliario dada por el MIDUVI (Ministerio de Desarrollo Urbano y Vivienda), así como el análisis acerca del manejo de la gestión de riesgos, donde en base a los parámetros considerados en las metodologías "Land Risk Management Model” y "Risk-Based Planning Aproach" se evaluó cual es la información territorial de utilidad para la gestión de riesgos y si esta información se encontraba disponible en los catastros inmobiliarios municipales. Para el diseño de la metodología se tomó como base el esquema dado en el proceso de gestión de riesgos de la AS/NZS ISO 31000:2009, además, de una adaptación a la realidad local, de las similitudes que tenían entre sí las dos metodologías tomadas como referencia. En la Figura 1 se observa más detalladamente el proceso seguido, a través del cual se interrelaciono los conceptos de administración territorial y de gestión de riesgos; una vez diseñada la metodología se procedió a implementarla en la zona de estudio y a partir de los resultados obtenidos se generó el SCR con ayuda del software STDM al definirse derechos, restricciones y responsabilidades que están involucrados en la gestión de riesgos, acorde al modelo LADM de la ISO 19152:2009.

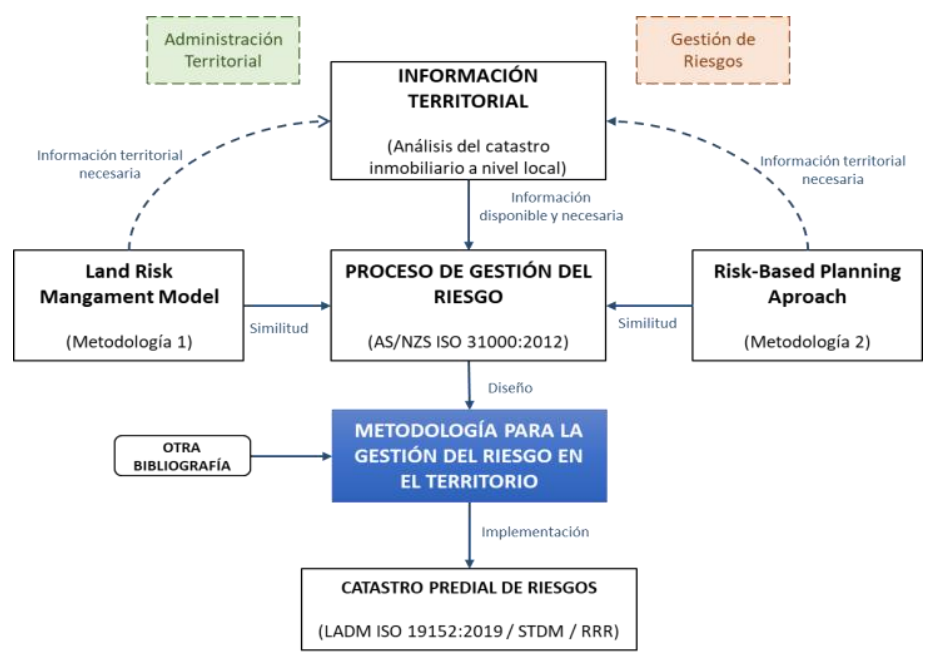

Figura 1. Proceso seguido en el diseño de la metodología

Revista Geoespacial (2019) 
La metodología propuesta, como muestra el modelo conceptual de la Figura 2, está constituida por tres elementos principales que son el contexto, el proceso de gestión del riesgo en el territorio y la planificación, además, de forma transversal a estos tres elementos se tiene dos sub elementos que son la comunicación y consulta; y el monitoreo y evaluación.

El primer elemento permite contextualizar el escenario en el que se va a trabajar, mediante la identificación del lugar territorial, los factores de riesgo y los interesados en la gestión de riesgos en el territorio; el segundo elemento establece el intercambio de información necesaria entre los sistemas de administración territorial y el proceso de gestión del riesgo del cual se obtendrá una evaluación del riesgo en relación al territorio; mientras el último elemento permite a los usuarios, a partir de la evaluación realizada, tomar las acciones necesarias dentro de la planificación territorial para reducir el riesgo. Los dos sub elementos transversales a todo el proceso: comunicación y consulta, y monitoreo y evaluación, asegura el óptimo desarrollo e implementación de la metodología.

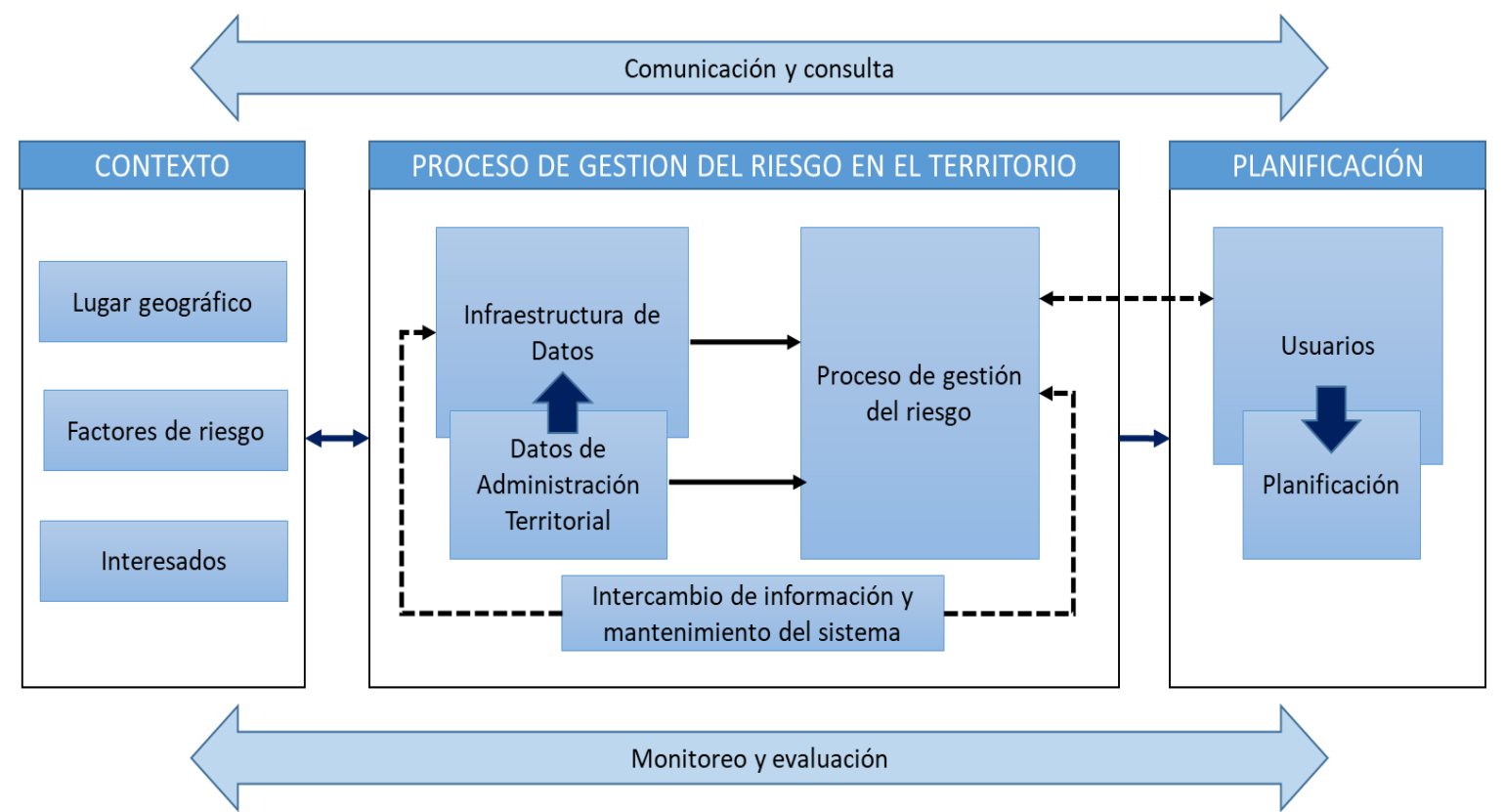

Figura 2. Modelo conceptual, metodología para la gestión de riesgos en el territorio Fuente: Adaptado de (Potts, 2013)

El proceso de gestión del riesgo intenta seguir lo planteado por Saunders, et al. (2013) en 'Risk-based land use planning for natural hazard risk reduction', lo cual a su vez está alineado con los pasos para la valoración de riesgos que se consideran dentro del proceso de gestión de riesgos en la AS/NZS ISO 31000:2009. Como muestra la Figura 3, se han considerado tres pasos fundamentales, los cuales corresponden a la identificación del riesgo, donde son determinadas las limitaciones, incertidumbres y asunciones; el análisis del riesgo, en función de una valoración de las posibles consecuencias y la probabilidad de ocurrencia del evento mediante el uso de una tabla multiriesgos y una escala de probabilidad de ocurrencia correspondientemente; como tercer paso está la evaluación del riesgo desde el ámbito territorial, donde se determinan el nivel de riesgo y su correspondiente estado de consentimiento. 


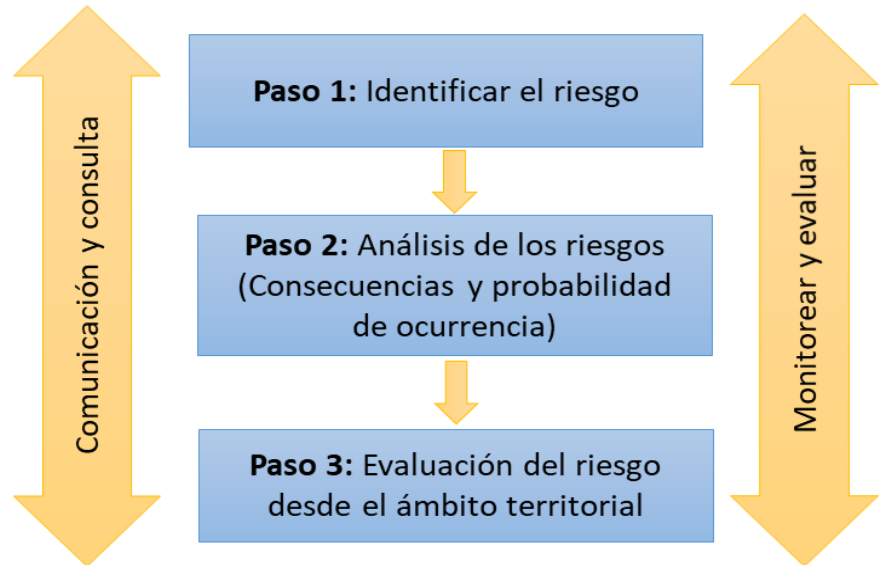

Figura 3. Pasos que conforman el proceso de gestión del riesgo Fuente: Adaptado de (Saunders, et al., 2013)

\section{IMPLEMENTACION DE LA METODOLOGÍA}

\section{Área de estudio}

La implementación de la metodología se llevó a cabo de forma piloto en parte de las probables áreas afectadas en el valle de Los Chillos por los lahares producto de una posible actividad eruptiva del volcán Cotopaxi, los cuales son flujos lodosos cargados de partículas sólidas de diferentes tamaños que se desplazan a grandes velocidades por las laderas del volcán debido a la fusión de la capa de hielo glacial que recubre la parte superior del cono que alcanza una altura de 5.897 metros sobre el nivel del mar, entre las principales poblaciones afectadas están la ciudad de Latacunga y el valle de Los Chillos, específicamente la conurbación DMQ GADMUR (Aguilera \& Toulkeridis, 2005). Los drenajes naturales que nacen en la cima del volcán sirven de camino para el desplazamiento de los lahares; así la afectación se extiende por las cuencas hidrográficas de los ríos Esmeraldas al norte, Pastaza al sur y Napo al este. En el caso del valle de Los Chillos, el área se ve afectada por los lahares transportados a través de los ríos Pita y Santa Clara que confluyen en el río San Pedro, y forman parte de la cuenca hidrográfica del río Esmeraldas. Uno de los modelamientos más recientes de los lahares que pueden afectar al valle de Los Chillos fue realizado en el año 2018 siguiendo la cuenca del río Pita, en el cual se determinó las áreas de inundación por el derretimiento de los glaciares, al ser uno de los datos más actuales se tomó esta como área de estudio, en el mapa de la Figura 5 se muestran los límites del área que abarca 373,36 ha y está distribuida entre el GADMUR y el DMQ que en conjunto a los actores privados y ciudadanos vinculados al área afectada son los principales afectados, la principal vulnerabilidad está relacionada con el crecimiento poblacional sin una adecuada planificación territorial que ha experimentado la zona. 


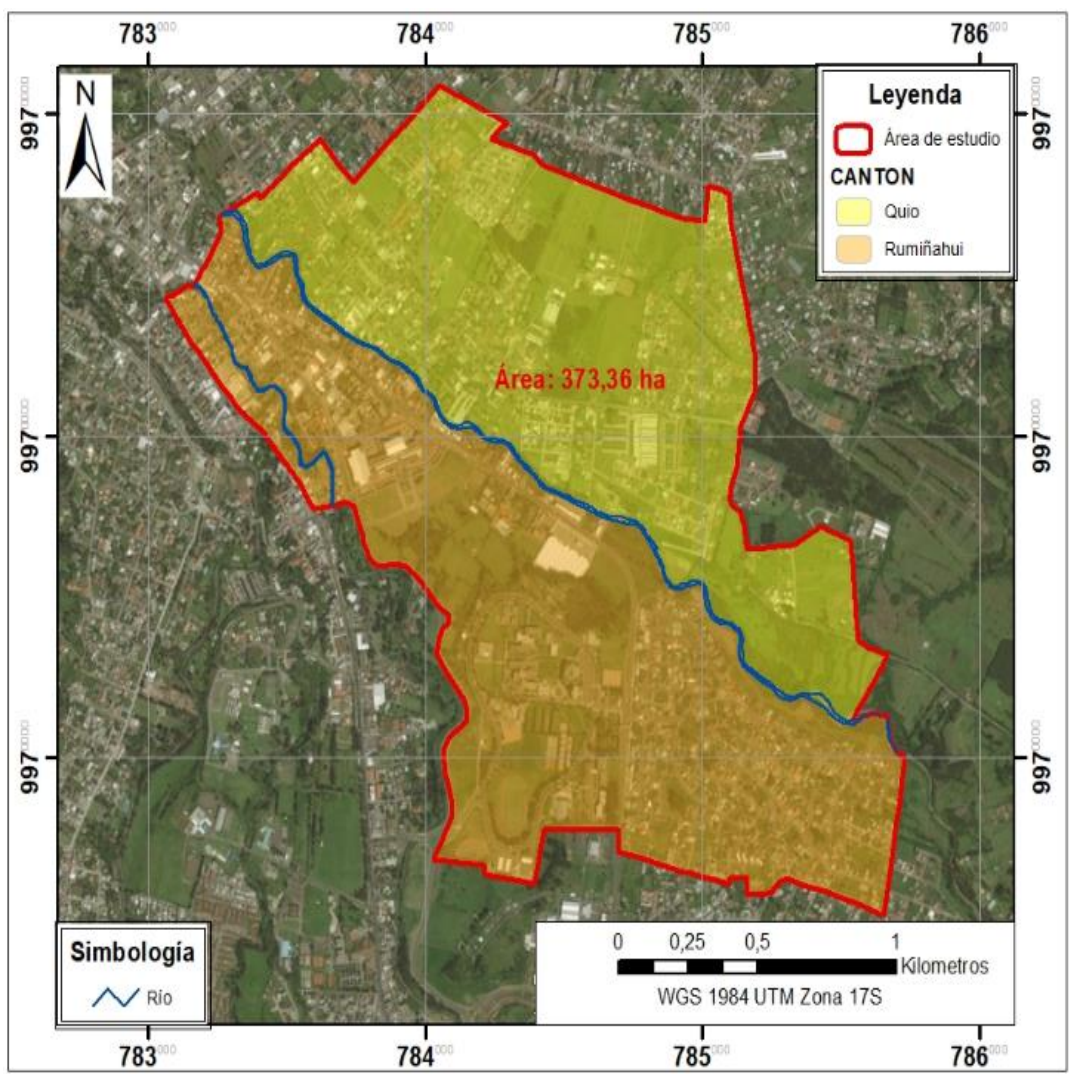

Figura 5. Área de estudio, localizada entre los cantones Quito y Rumiñahui

\section{Identificación del riesgo}

El área de impacto directo del lahar dentro de la zona de estudio abarca 239,52 ha, donde la altura de la columna del lahar va desde los 0,005 $\mathrm{m}$ hasta los 17,726 m, adicionalmente se consideró una zona de seguridad de 50m alrededor de la zona de impacto, esto sigue el criterio tomado por Padilla (2017) en el cálculo de tiempos de evacuación ante una eventual erupción del volcán Cotopaxi. El área como se ve en el mapa de la Figura 4, fue dividida en cuatro zonas de valoración: las zonas 1 y 2 consideran las áreas de impacto del lahar con una columna $\geq 2,50$ $\mathrm{m}$ de altura, mientras las zonas 3 y 4 corresponden a la suma de las áreas de impacto con una columna $<2,50 \mathrm{~m}$ y la franja de seguridad de $50 \mathrm{~m}$, de igual forma se consideró el límite político administrativo entre el DMQ y el GADMUR, así las zonas 4 y 1 se ubican en el DMQ mientras las zonas 3 y 2 en el GADMUR. El valor de 2,50 metros está basado en la altura promedio de una planta de construcción; al existir en su mayoría viviendas de dos plantas en la zona, se consideró la viabilidad de una evacuación vertical. 


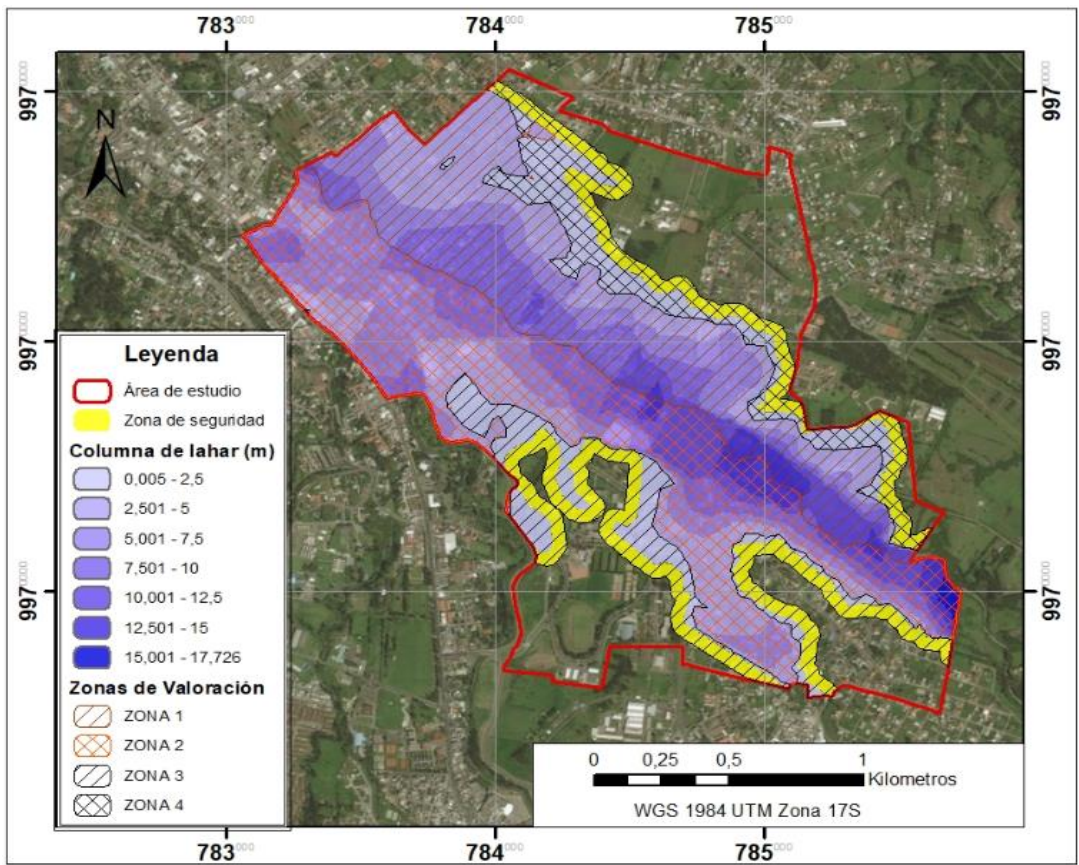

Figura 4. Lahar, área de impacto y zonificaciones para la valoración de riesgos

Las asunciones, limitaciones e incertidumbres en las cuales se basó el proceso de la gestión de riesgos en este estudio, son enlistados en la Tabla 1.

Tabla 1. Asunciones, limitaciones e incertidumbres consideradas en la aplicación del proceso

\begin{tabular}{|c|c|}
\hline Asun & $\begin{array}{l}\text { Se asume una erupción similar a la de 1877, en la cual se produjo el } \\
\text { deshielo del glacial del volcán Cotopaxi. } \\
>\quad \text { Se considera únicamente la población fija, no la población móvil. } \\
\text { Las viviendas de más de dos plantas son lo suficientemente resistentes al } \\
\text { paso del lahar. }\end{array}$ \\
\hline Lim & $\begin{array}{l}\text { ue llegaría por la } \\
\text { udio. }\end{array}$ \\
\hline Incertidumbres & $\begin{array}{l}\text { Complicaciones que podrían ocasionar otras amenazas volcánicas como } \\
\text { la caída de ceniza. } \\
\text { Efectos que ocasionarían condiciones climáticas extremas como lluvias } \\
\text { torrenciales que pudieran ocurrir a la par de la erupción. }\end{array}$ \\
\hline
\end{tabular}

Determinación de consecuencias

La determinación de las consecuencias se realizó en función de los parámetros: infraestructura - edificaciones, económico y de salud y seguridad, establecidos dentro de la tabla multi-riesgos. El parámetro de infraestructura abarcó la evaluación de construcciones, construcciones de uso social/cultural, instalaciones críticas y servicios vitales, para identificar los tres primeros se depuraron, estandarizaron e integraron los catastros inmobiliarios municipales del GADMUR y GAD DMQ, posteriormente el catastro fue actualizado mediante la digitalización de una ortofoto del año 2018. La identificación de las construcciones de tipo social/cultural e instalaciones críticas se realizó con ayuda de la información acerca de uso del suelo y de construcciones, contenida en las fichas catastrales, la información fue corroborada mediante visitas de campo. Finalmente fueron contabilizadas las construcciones funcionalmente comprometidas y calculados los porcentajes de las mismas en las cuatro zonas 
de valoración y para cada uno de los tipos de construcción, las construcciones consideradas funcionalmente comprometidas son aquellas localizadas en áreas donde la columna de lahar fuera $>0,5 \mathrm{~m}$.

Dentro de lo correspondiente a servicios vitales fueron evaluados los servicios de agua potable, alcantarillado, electricidad y de transporte, la evaluación abarco al total de la población urbana afectada en el GADMUR y el GAD DMQ. El servicio de agua potable está a cargo de la Empresa Pública Metropolitana de Agua Potable y Saneamiento (EPMAPS) en Quito y por la Dirección de Agua Potable y Alcantarillado en Rumiñahui, se encontró que los sistemas de abastecimiento se encuentran conformados por elementos de captación, tratamiento y distribución del agua. En ambas áreas urbanas la principal afectación se da en las líneas de captación ya que pese a las medidas de mitigación tomadas, en el caso de Quito, de las 9 zonas hídricas con las que cuenta la ciudad se vería especialmente afectada la zona hídrica PuengasíPlacer, al abastecerse con líquido proveniente del río Pita, lo que limitaría el servicio en el sur y centro sur de la ciudad de Quito afectando al 32,79 \% de la población urbana hasta la reparación de la línea que tomaría cerca de 8 meses (Peña, 2016). Mientras tanto el cantón Rumiñahui se abastece de 17 vertientes y pozos, su principal sistema de captación es el de Molinuco que abastece al 50\% de la población del cantón se vería especialmente afectado por el paso del lahar ya que la tubería cruza por debajo del río, lo cual generaría un importante desabastecimiento a la mitad de la población hasta reparar los desperfectos, lo que podría llevar más de un mes.

La distribución de energía eléctrica de los cantones Quito y Rumiñahui está a cargo de la Empresa Eléctrica Quito (EEQ), el sistema eléctrico está conformado por las subestaciones de transmisión y distribución, las líneas de subtransmisión, las líneas de distribución y los puntos de carga (medidores), y es abastecido principalmente por el Sistema Nacional de Transmisión (SNT) y en menor medida de pequeñas centrales hidroeléctricas y térmicas, propiedad de la EEQ. Tras realizar el análisis se determinó que la principal afectación sería debido a la pérdida de las líneas de distribución (postes y cableado) en la zona de afectación directa del lahar y de la subestación de San Rafael. El tiempo de reposición se estimó en un lapso no menor a 3 meses en base a tiempos estimados para la reposición de subestaciones en condiciones normales (Narváez, 2007). En lo referente a transporte, luego de una revisión de estudios anteriores en los que se analizó las afectaciones a la red vial y se determinó las afectaciones por el lahar en el valle de Los Chillos, el tiempo estimado de reposición y la incidencia que tendría en el servicio se consideró una vulnerabilidad baja de la red vial ante la ocurrencia de un lahar del Cotopaxi (Narváez, 2007; Tibanlombo y Villacís, 2013). Así la principal afectación del transporte se daría en la zona de afectación directa del lahar y de la zona inter lahar, por más de dos meses hasta el despeje de las vías principales. De igual forma el sistema de saneamiento se vería principalmente afectado por el taponamiento de tuberías y desagües debido a la acumulación de material, el tiempo de paralización de este servicio en el área afectada sería indefinido.

En la estimación del porcentaje de la población urbana del DMQ y del GADMUR que se quedaría sin los servicios de electricidad, alcantarillado y transporte se consideró una zona adicional de $50 \mathrm{~m}$ alrededor del lahar, Figura 5, además, se incluyó dentro del área afectada por el lahar a la zona inter lahar que se localiza entre los ríos Santa Clara y Pita, representada de color naranja. El número de afectados se obtuvo al cruzar un mapa de densidad poblacional al año 2019 con las zonas de valoración. 


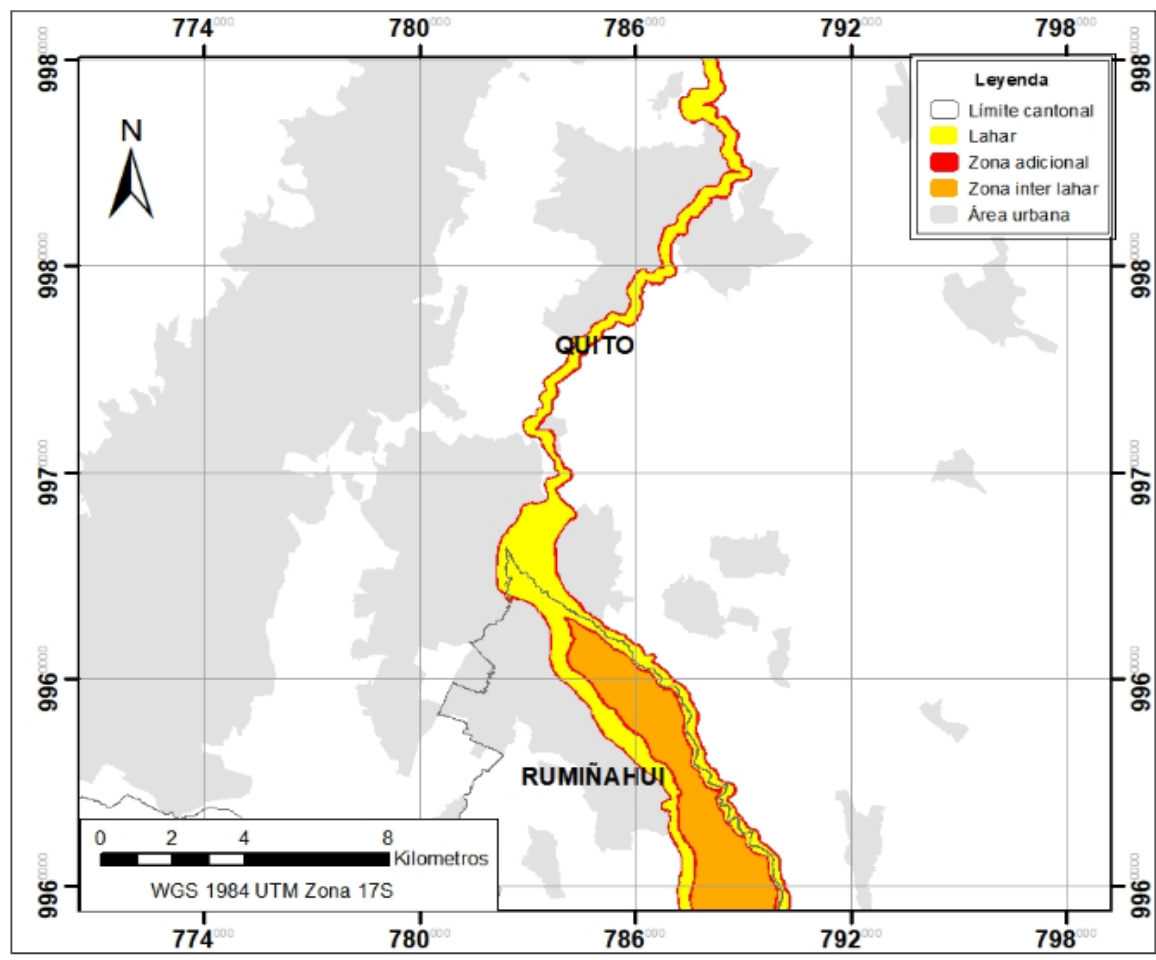

Figura 5. Afectación del lahar en las áreas urbanas del GADMUR y DMQ

En lo referente al parámetro de salud y seguridad, se estimó el número de posibles muertes debido a la ocurrencia del lahar, a partir de un escenario propuesto en el que las personas que no se encuentran preparadas para afrontar una situación de emergencia producto del lahar del Cotopaxi, pondrían en peligro su integridad física al no evacuar a tiempo; fueron consideradas únicamente las zonas 1 y 2, al ser las de mayor peligro. Para estimar el grado de preparación de la población se realizó una encuesta de selección múltiple, en la cual se preguntó a las personas si tenían conocimiento que el lugar donde se encontraban es zona de peligro por lahares, si habían participado en simulacros de evacuación por el lahar del Cotopaxi en el pasado, si conocían las rutas de evacuación y las zonas seguras, además, se preguntó qué tiempo consideraban como óptimo para evacuar el área en caso de emergencia y cuán probable creían experimentar una erupción del volcán Cotopaxi en el transcurso de su vida, la encuesta fue aplicada a una muestra de 352 personas con un nivel de confianza del $95 \%$ y una población conocida de 4177 habitantes en las zonas 1 y 2 . La encuesta fue realizada con ayuda de la aplicación móvil "ODK Collect" y mostró que el 68,10 \% de la población que habita en las zonas 1 y 2 estaría preparada o tiene conocimientos básicos de qué hacer en el caso de una emergencia, mientras el $31,90 \%$ no se encuentra preparada, el número estimado de muertes fue calculado para cada zona de valoración en base a los porcentajes obtenidos.

Para el cálculo de las pérdidas económicas se siguió la ecuación (1), en lugar del PIB se utilizó el VAB (Valor Agregado Bruto), el cual equivale a los valores de bienes y servicios generados en una región, y es un valor representativo similar al PIB.

$$
P I B k=\frac{S U * C S U+C U * C C U+E M * E C M}{P I B C}
$$


Donde:

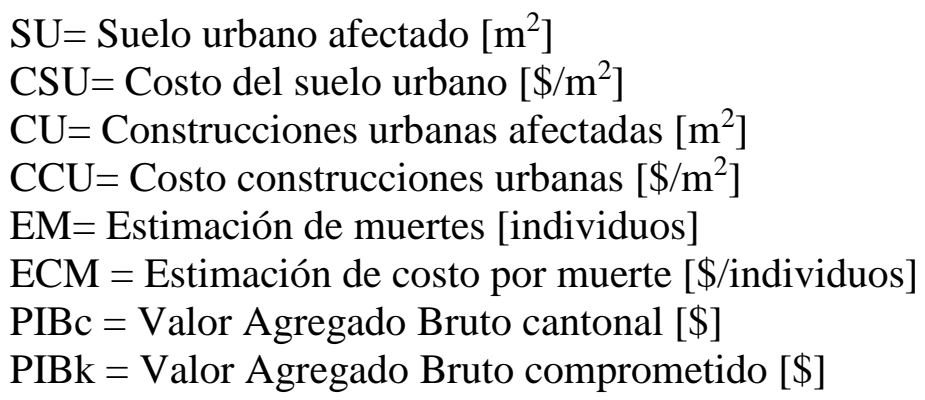

Los valores usados de costo del suelo y de construcción fueron tomados del estudio "Economic risk assessment of Cotopaxi volcano, Ecuador, in case of a future lahar emplacement" realizado por Rodríguez, et al. (2016), donde se establecieron los valores de $\$ 250$ por $\mathrm{m}^{2}$ de suelo urbano y 500 por $\mathrm{m}^{2}$ de construcción urbana para el valle de Los Chillos. Los valores para los VAB cantonales, que equivalen a los valores de bienes y servicios generados en cada cantón, fueron obtenidos de los valores agregados brutos cantonales provisionales dados por el Banco Central del Ecuador para el año 2017. La estimación de costo por muerte (ECM) fue calculada de acuerdo a los años de vida como personas económicamente activas que se perderían por muerte, 31 años; y el VABpi (Valor agregado bruto por habitante), la Tabla 2 muestra el ECM para cada cantón.

Tabla 2. Estimación costo económico por muerte (ECM)

\begin{tabular}{ccccc}
\hline Cantón & Habitantes & VABc (M.\$) & VABpi (\$) & $\begin{array}{c}\text { ECM } \\
\text { (\$individuo) }\end{array}$ \\
\hline Quito & 2.644 .145 & $24.426,598$ & $9.237,99$ & $286.377,84$ \\
\hline Rumiñahui & 107.043 & 803,979 & $7.510,80$ & $232.834,93$ \\
\hline
\end{tabular}

La probabilidad de ocurrencia se determinó acorde al tipo de actividad y los períodos de descanso identificados en los registros históricos del volcán Cotopaxi de los últimos 2000 años, que indican como tiempo estimado que ha transcurrido entre dos erupciones sucesivas es de $117 \pm 70$ años, con la consideración de que la última erupción fue en 1877 y que el tiempo de reposo hasta el 2019 ha sido de 142 años, se esperaría una erupción dentro de los siguientes 45 años (Aguilera \& Toulkeridis, 2005). Al esperarse la próxima erupción dentro de un lapso menor a 50 años acorde a la tabla de probabilidad de ocurrencia, la probabilidad de una eventual erupción del volcán Cotopaxi corresponde a un nivel 5, el más alto, es decir probable.

\section{Evaluación del riesgo desde el ámbito territorial}

Una vez determinadas las consecuencias y la probabilidad de ocurrencia se determinó el nivel de riesgo y el estado de consentimiento para cada una de las zonas, mediante el cuadro de la Figura 6. La gestión de riesgos es evaluada ante el escenario posible, por lo cual se tomó la consecuencia más catastrófica, es decir con el nivel más alto de severidad en cada una de las zonas; mientras la probabilidad de ocurrencia del evento para todas las zonas de valoración se mantuvo como "probable". 


\begin{tabular}{|c|c|c|c|c|c|c|c|}
\hline & \multicolumn{5}{|c|}{ CONSECUENCIAS } & \multirow{2}{*}{ Nivel de riesgo } & \multirow{2}{*}{ Consentimiento } \\
\hline PROBABILIDAD & I & II & III & IV & V & & \\
\hline 5 & & & & & & Bajo & Permitido \\
\hline 4 & & & & & & Moderado & Controlado \\
\hline $\begin{array}{l}3 \\
2\end{array}$ & & & & & & Alto & Discrecional \\
\hline 1 & & & & & & Extremo & No conformidad \\
\hline
\end{tabular}

Figura 6. Evaluación del riesgo desde el ámbito territorial para el lahar

\section{Recomendaciones de planificación territorial}

Fueron propuestas una serie de recomendaciones en relación a la planificación territorial, mismas que siguen el proceso para el tratamiento del riesgo que implica tomar acciones dirigidas a evitar, reducir, trasladar o conservar el riesgo, las cuales deben ser aplicadas en ese orden según sea posible su ejecución. Las acciones para tratar el riesgo fueron divididas en dos categorías, aquellas enfocadas en reducir el riesgo en las zonas de valoración y alcanzan una aplicación a nivel predial; y aquellas que si bien reducen el riesgo en las zonas de valoración también reducen el riesgo del resto de la población en ambas ciudades y su aplicación está a nivel del cantón. Estas acciones estuvieron dirigidas a tres áreas de aplicación (AP) que comparten características comunes dentro de la zona de estudio y van enfocadas principalmente a disminuir el número de construcciones de uso particular que se verían comprometidas por el lahar a través de una reubicación; el trasladar y evitar que construcciones que brindan servicios de importancia social, cultural o instalaciones críticas se ubiquen en zonas de afectación y el aseguramiento de los bienes que podrían ser afectados para evitar pérdidas económicas. Además, se asignó el nivel de riesgo correspondiente a cada uno de los predios afectados, donde las zonas 1 y 2 conservaron un nivel de riesgo "Extremo" y un estado de consentimiento de "No conformidad", mientras por las características de las zonas 3 y 4 , y las acciones propuestas el nivel de riesgo bajo a "Alto" y un estado de consentimiento "Discrecional".

\section{GENERACIÓN DEL SISTEMA CATASTRAL DE RIESGOS}

Con la finalidad de integrar información del catastro inmobiliario convencional con información referente a la gestión de riesgos naturales, fue desarrollado el SCR que permite registrar los peligros naturales a los cuales se encuentran expuestas las diferentes unidades espaciales, la valoración del riesgo y las acciones a tomar para tratarlo. Esto no solo ayuda a asentar la información generada sobre riesgos, sino que permite transmitirla a los interesados, entre los cuales se encuentran la ciudadanía, gobiernos locales, gobierno nacional y diferentes organizaciones e instituciones vinculadas ya sea a la administración territorial o la gestión del riesgo, y así puedan tomar las acciones necesarias. El SCR aplica el modelo conceptual de la LADM descrito en la ISO 19152:2012, donde la gestión de riesgos implica una serie de nuevos derechos, restricciones y responsabilidades (RRR por sus siglas en inglés) sobre el predio y sobre quien ejerce la tenencia del mismo, En la Figura 7 se muestra la clasificación de los derechos, restricciones y responsabilidades dados por la gestión de riesgos acorde al esquema de la LADM. 


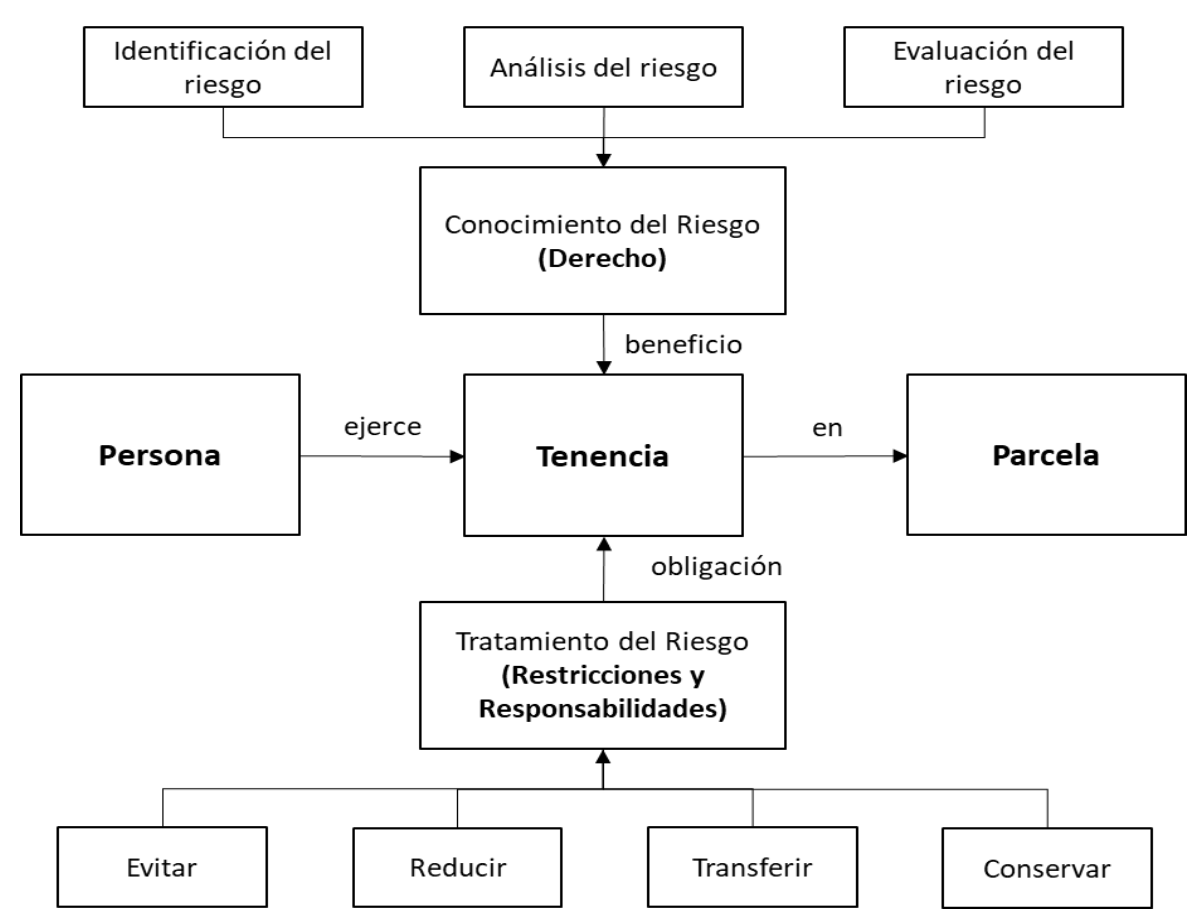

Figura 7. Derechos, restricciones y responsabilidades ligados a la gestión de riesgos

Los derechos, restricciones y responsabilidades varían acorde al nivel territorial al cual está dirigido, en este caso el sistema catastral se encuentra dirigido al nivel predial. La información de riesgos que fue ingresada al sistema catastral se detalló dentro de la ficha catastral de riesgos acorde al área de aplicación (AP) en la que se encuentra el predio, una vez definida la estructura del sistema catastral y la información que sería ingresada, se procedió a su creación con la ayuda del software STDM; fueron generados informes automáticos para cada predio, los cuales poseen un mapa de ubicación del predio, información del predio, del propietario y de la evaluación de riesgos.

\section{RESULTADOS Y DISCUSIÓN}

\section{DATOS GENERADOS}

\section{Valoración del riesgo}

Una vez determinadas las consecuencias en lo referente a construcciones, construcciones con interés social/cultural, instalaciones críticas, ámbito económico y de salud y seguridad, y aplicar la tabla multi-riesgos para determinar el nivel de consecuencias de cada uno de estos elementos, se obtuvo la severidad del impacto para cada una de las zonas de valoración como muestra la Tabla 3, donde se observa que todas las zonas de valoración resultaron con al menos un parámetro catalogado como catastrófico, sin embargo, es evidente que las principales afectaciones se dan en las zonas 1 y 2 , y están especialmente ligadas al alto número de construcciones existentes en la zona de riesgo. 
Tabla 3. Severidad del impacto por el lahar en las zonas de valoración

\begin{tabular}{|c|c|c|}
\hline $\begin{array}{c}\text { Zona de } \\
\text { valoración }\end{array}$ & Consecuencias & Severidad del impacto \\
\hline \multirow{5}{*}{ ZONA 1} & Social/Cultural & $\begin{array}{l}\text { Catastrófico - Todos los ESC están funcionalmente } \\
\text { comprometidos. }\end{array}$ \\
\hline & Construcciones & $\begin{array}{l}\text { Catastrófico - Todos los edificios están funcionalmente } \\
\text { comprometidos. }\end{array}$ \\
\hline & $\begin{array}{c}\text { Instalaciones } \\
\text { críticas }\end{array}$ & Insignificante - No existen IC. \\
\hline & Económico & Mayor - 1,87 \% del PIB del cantón Quito. \\
\hline & Salud y Seguridad & Catastrófico - 596 muertos, no heridos. \\
\hline \multirow{5}{*}{ ZONA 2} & Social/Cultural & $\begin{array}{l}\text { Catastrófico - Todos los ESC están funcionalmente } \\
\text { comprometidos. }\end{array}$ \\
\hline & Construcciones & $\begin{array}{l}\text { Catastrófico - Todos los edificios están funcionalmente } \\
\text { comprometidos. }\end{array}$ \\
\hline & $\begin{array}{l}\text { Instalaciones } \\
\text { críticas }\end{array}$ & Insignificante - No existen IC. \\
\hline & Económico & Catastrófico - 74,35 \% del PIB del cantón Rumiñahui. \\
\hline & Salud y Seguridad & Catastrófico - 738 muertos, no heridos. \\
\hline \multirow{5}{*}{ ZONA 3} & Social/Cultural & $\begin{array}{l}\text { Catastrófico - 26,32\% de los ESC están funcionalmente } \\
\text { comprometidos. }\end{array}$ \\
\hline & Construcciones & $\begin{array}{l}\text { Catastrófico }-25 \% \text { de los edificios están funcionalmente } \\
\text { comprometidos. }\end{array}$ \\
\hline & $\begin{array}{l}\text { Instalaciones } \\
\text { críticas }\end{array}$ & Insignificante - No existen IC. \\
\hline & Económico & Mayor $-6,01 \%$ del PIB del cantón Rumiñahui. \\
\hline & Salud y Seguridad & Insignificante - No muertos, no heridos. \\
\hline \multirow{5}{*}{ ZONA 4} & Social/Cultural & Insignificante - No existen ESC. \\
\hline & Construcciones & $\begin{array}{l}\text { Catastrófico - } 56 \% \text { de los edificios están funcionalmente } \\
\text { comprometidos. }\end{array}$ \\
\hline & $\begin{array}{l}\text { Instalaciones } \\
\text { críticas }\end{array}$ & Insignificante - No existen IC. \\
\hline & Económico & Moderado - 0,28\% del PIB del cantón Quito. \\
\hline & Salud y Seguridad & Insignificante - No muertos, no heridos. \\
\hline
\end{tabular}

$\mathrm{ESC}=$ Edificio con significado social/cultural; $\mathrm{IC}=$ Infraestructura crítica; FS= Fuera de servicio; PPCA= Población del pueblo o ciudad afectada; Funcionalmente comprometido= el edificio no puede ser usado para su propósito inmediatamente después del evento.

El nivel de consecuencias en los servicios vitales fue evaluado por separado ya que la afectación a estos servicios se cuantifica a nivel de toda la población y no únicamente dentro de las zonas de valoración. Tras aplicar la tabla multi-riesgos la severidad del impacto para las poblaciones urbanas del GADMUR y el DMQ, los porcentajes de población urbana afectada por el desabastecimiento o paralización de cada servicio es indicado en la Tabla 4, siendo la principal afectación en lo correspondiente al abastecimiento de agua potable, especialmente para el GADMUR. 
Tabla 4. Severidad del impacto por el lahar en la población urbana

\begin{tabular}{|c|c|c|}
\hline Población & Consecuencias & Severidad del impacto \\
\hline \multirow{4}{*}{ DMQ } & Agua potable & $\begin{array}{l}\text { Catastrófico - El } 32,79 \% \text { de la población urbana tendría } \\
\text { racionamientos por al menos } 8 \text { meses. }\end{array}$ \\
\hline & Alcantarillado & $\begin{array}{l}\text { Insignificante }- \text { El } 0,64 \% \text { de la población urbana no tendría } \\
\text { el servicio por tiempo indefinido. }\end{array}$ \\
\hline & Electricidad & $\begin{array}{l}\text { Insignificante - El 0,64 \% de la población urbana no tendría } \\
\text { el servicio por al menos } 3 \text { meses. }\end{array}$ \\
\hline & Transporte & $\begin{array}{l}\text { Insignificante - El 0,64 \% de la población urbana tendría un } \\
\text { servicio limitado por al menos } 2 \text { meses. }\end{array}$ \\
\hline \multirow{4}{*}{ GADMUR } & Agua potable & $\begin{array}{l}\text { Catastrófico - El } 50 \% \text { de la población urbana tendría } \\
\text { racionamientos por más de un mes. }\end{array}$ \\
\hline & Alcantarillado & $\begin{array}{l}\text { Catastrófico - El } 29,16 \% \text { de la población urbana no tendría } \\
\text { el servicio por tiempo indefinido. }\end{array}$ \\
\hline & Electricidad & $\begin{array}{l}\text { Catastrófico - El } 29,16 \% \text { de la población urbana no tendría } \\
\text { el servicio por al menos } 3 \text { meses. }\end{array}$ \\
\hline & Transporte & $\begin{array}{l}\text { Catastrófico - El 29,16\% de la población urbana tendría un } \\
\text { servicio limitado por al menos } 2 \text { meses. }\end{array}$ \\
\hline
\end{tabular}

\section{Tratamiento del riesgo}

En los mapas de la Figura 8, se muestran los predios localizados en cada una de las tres áreas de aplicación, así como los niveles de riesgos asignados a los mismos. Se observa que los predios más cercanos al río, como se ve de color rojo en el mapa de la derecha son los más vulnerables y por consiguiente las acciones recomendadas en las AP 1 y AP 2 son más estrictas que las del AP 3.
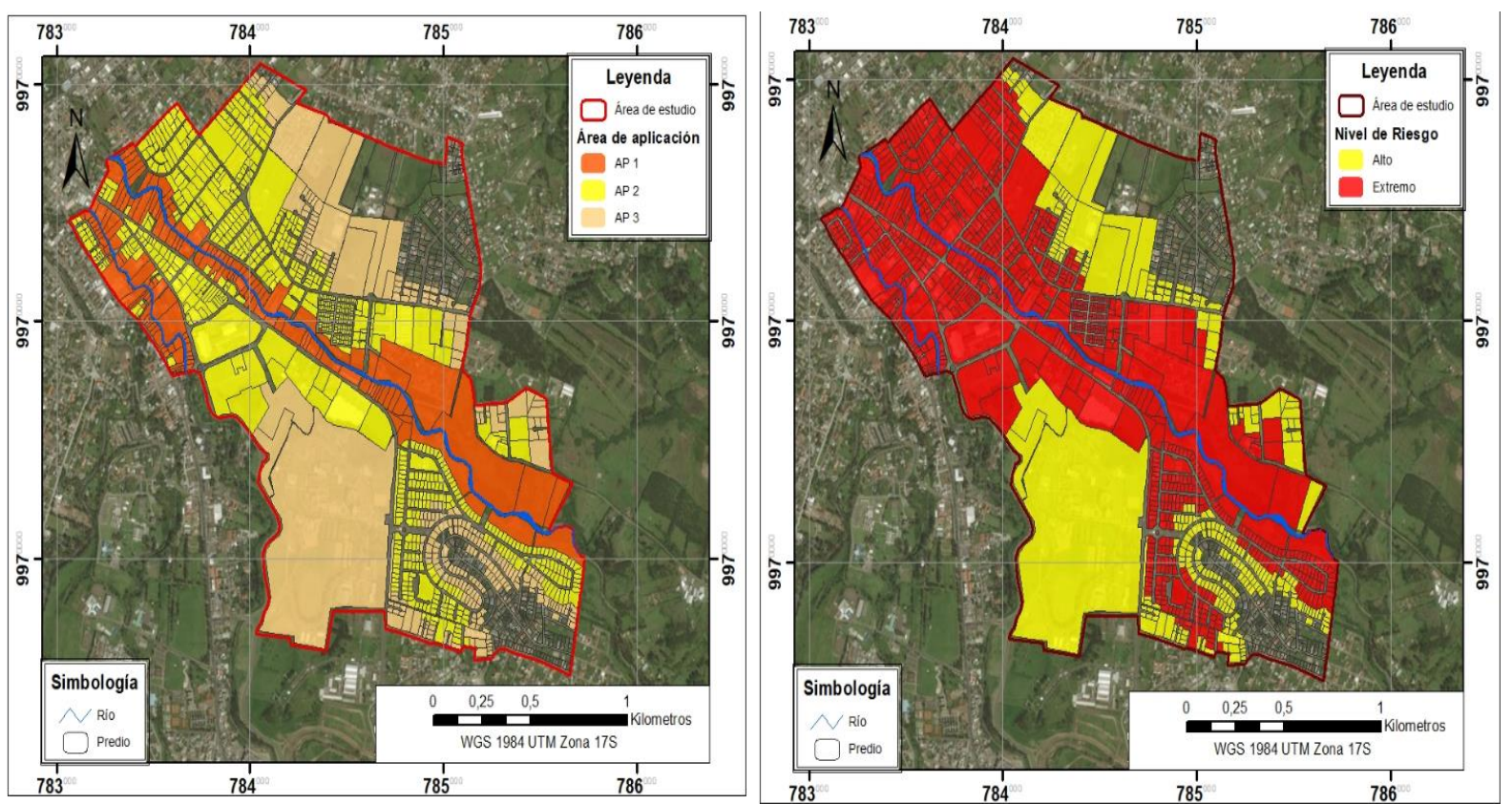

Figura 8. Áreas de aplicación delimitadas a nivel predial (izq.), asignación de los niveles de riesgo a los predios (der.)

Revista GEOESPACIAL (2019) 
En la Tabla 5 están enlistadas las acciones que deberían ser implementadas para tratar el riesgo en cada zona de valoración y el número de predios dentro de cada área de aplicación.

Tabla 5. Acciones específicas para las zonas de valoración y su área de aplicación

\begin{tabular}{|c|c|c|c|c|}
\hline $\begin{array}{l}\text { Zona de } \\
\text { valoración }\end{array}$ & Acciones & $\begin{array}{l}\text { Área de } \\
\text { aplicación } \\
\text { (AP) }\end{array}$ & $\begin{array}{l}\text { Extensión del } \\
\text { AP }\end{array}$ & Predios \\
\hline \multirow{5}{*}{$\begin{array}{l}\text { Zona } 1 \text { y } \\
\text { Zona } 2\end{array}$} & $\begin{array}{l}\text { Reubicación de las personas } \\
\text { asentadas justo a las riberas del río } \\
\text { para disminuir la cantidad de } \\
\text { construcciones afectadas. }\end{array}$ & AP 1 & $\begin{array}{l}\text { Predios ubicados } \\
\text { a una distancia de } \\
\text { hasta } 30 \mathrm{~m} \text { de la } \\
\text { ribera del río, se } \\
\text { incluyen predios } \\
\text { municipales } \\
\text { cercanos. }\end{array}$ & 164 \\
\hline & $\begin{array}{l}\text { No autorizar construcciones en } \\
\text { lotes vacíos y nuevas }\end{array}$ & \multirow{4}{*}{ AP 2} & \multirow{4}{*}{$\begin{array}{l}\text { Predios entre el } \\
\text { área AP1 y los } \\
\text { límites de las } \\
\text { zonas } 1 \text { y } 2 .\end{array}$} & \multirow{4}{*}{726} \\
\hline & $\begin{array}{l}\begin{array}{l}\text { construcciones en lotes } \\
\text { ocupados. }\end{array} \\
\text { ocua }\end{array}$ & & & \\
\hline & $\begin{array}{l}\text { No permitir la construcción de } \\
\text { nuevas IC y CSC. }\end{array}$ & & & \\
\hline & $\begin{array}{l}\text { Reubicar en áreas seguras los } \\
\text { edificios de uso socio cultural. }\end{array}$ & & & \\
\hline \multirow{3}{*}{$\begin{array}{l}\text { Zona } 3 \text { y } \\
\text { Zona } 4\end{array}$} & $\begin{array}{l}\text { Reubicar en áreas seguras los } \\
\text { edificios de uso socio cultural. }\end{array}$ & \multirow{3}{*}{ AP 3} & \multirow{3}{*}{$\begin{array}{l}\text { Predios } \\
\text { pertenecientes a } \\
\text { las zonas } 3 \text { y } 4\end{array}$} & \multirow{3}{*}{269} \\
\hline & $\begin{array}{l}\text { No permitir la construcción de } \\
\text { nuevas IC y CSC. }\end{array}$ & & & \\
\hline & $\begin{array}{l}\text { Edificios en esta zona deben tener } \\
\text { por lo menos dos plantas y deben } \\
\text { cumplir especificaciones técnicas } \\
\text { dadas por la autoridad }\end{array}$ & & & \\
\hline
\end{tabular}

En lo correspondiente a servicios vitales, la planificación que permita mitigar la severidad del impacto implica la toma de acciones por parte de los municipios y de las empresas o instituciones encargadas de brindar el servicio, siendo la principal afectación en lo correspondiente al abastecimiento de agua potable, especialmente para el GADMUR.

\section{FICHA CATASTRAL DE RIESGOS}

De la generación del SCR se obtuvo el modelo conceptual que permite la relación entre información generada por la administración territorial con los datos resultantes del proceso de gestión de riesgos, lo cual se ve reflejado en la Tabla 6, donde se visualiza la ficha catastral de riesgos, misma que indica la estructura e información contenida en la base datos administrada por el SCR y su relación con cada una de las cuatro clases básicas del modelo LADM, cuyos datos son tomados de los catastros inmobiliarios municipales y el resultado de la valoración del riesgo; ítems como la clave catastral sirven de enlace con las otras entidades de la base catastral municipal, mientras los referentes a ubicación y tenencia del predio, ayudan a mejorar la gestión de riesgos. Además, para conocer de la importancia de poner a disposición de todos los interesados la información relacionada a riesgos a través de infraestructuras tecnológicas 
territoriales como el catastro, en la encuesta realizada se incluyó una pregunta adicional donde los habitantes señalaron qué tan importante consideran que el gobierno local les proporcione información de los riesgos a los que están expuestas las propiedades en las que habitan o desarrollan sus actividades, a lo que el $94 \%$ de los encuestados respondió como muy importante.

Tabla 6. Detalle de la información incluida en la ficha catastral y la base de datos del SCR; y su relación con las principales clases de la LADM.

\begin{tabular}{|c|c|c|c|c|c|}
\hline \multirow{2}{*}{\multicolumn{2}{|c|}{ Información }} & \multicolumn{3}{|c|}{ Área de aplicación } & \multirow{2}{*}{ Clases LADM } \\
\hline & & AP 1 & AP 2 & \multirow[t]{2}{*}{ AP 3} & \\
\hline \multirow{6}{*}{ 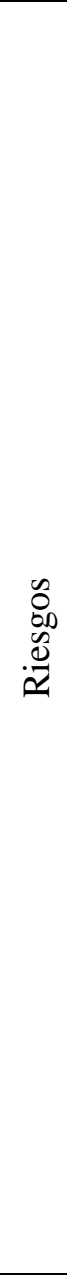 } & Peligro & & Lahar & & \multirow{4}{*}{$\begin{array}{c}\text { LA_RRR } \\
\text { (Derechos) }\end{array}$} \\
\hline & Posibilidad & \multicolumn{3}{|c|}{ Posible } & \\
\hline & Consecuencias & \multicolumn{3}{|c|}{ Catastrófico } & \\
\hline & $\begin{array}{l}\text { Nivel de } \\
\text { Riesgo }\end{array}$ & \multicolumn{2}{|c|}{ Extremo } & Alto & \\
\hline & $\begin{array}{l}\text { Acción } \\
\text { requerida }\end{array}$ & \multicolumn{2}{|c|}{$\begin{array}{l}\text { Plan de tratamiento debería } \\
\text { ser desarrollado, } \\
\text { implementado y evaluado } \\
\text { anualmente }\end{array}$} & $\begin{array}{l}\text { Acción inmediata } \\
\text { es requerida, la } \\
\text { implementación } \\
\text { del tratamiento } \\
\text { debe ser } \\
\text { extensiva y } \\
\text { evaluada } \\
\text { regularmente }\end{array}$ & \\
\hline & Tratamiento & $\begin{array}{l}\text { 1) } \\
\text { Reubicación } \\
\text { a zonas } \\
\text { seguras }\end{array}$ & $\begin{array}{l}\text { 1) No se } \\
\text { permiten } \\
\text { nuevas } \\
\text { construcciones } \\
\text { 2) Seguro } \\
\text { contra riesgos } \\
\text { obligatorio }\end{array}$ & $\begin{array}{l}\text { 1) No se } \\
\text { permiten } \\
\text { construcciones } \\
\text { con fines social, } \\
\text { cultural o } \\
\text { instalaciones } \\
\text { críticas 2) } \\
\text { Construcciones } \\
\text { particulares } \\
\text { deben cumplir } \\
\text { características } \\
\text { dadas por la } \\
\text { autoridad 3) } \\
\text { Seguro contra } \\
\text { riesgos opcional }\end{array}$ & $\begin{array}{c}\text { LA_RRR } \\
\text { (Restricciones y } \\
\text { Responsabilidades) }\end{array}$ \\
\hline \multirow{6}{*}{ 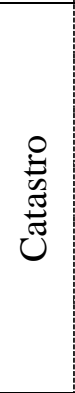 } & \multirow{3}{*}{ Ubicación } & \multicolumn{3}{|c|}{ Clave catastral* } & \multirow{4}{*}{ LA_SpatialUnit } \\
\hline & & & Cantón* & & \\
\hline & & & Dirección* & & \\
\hline & \multirow{2}{*}{$\begin{array}{l}\text { Tenencia } \\
\text { aspectos } \\
\text { legales }\end{array}$} & \multicolumn{3}{|c|}{ Régimen de tenencia* } & \\
\hline & & \multicolumn{3}{|c|}{ Propietario* } & LA_Party \\
\hline & $\begin{array}{c}\text { Objeto } \\
\text { catastral }\end{array}$ & \multicolumn{3}{|c|}{ Predio** } & LA_BaUnit \\
\hline
\end{tabular}

* El dato cambia según la base catastral inmobiliaria, **Indica la unidad espacial en la que se está trabajando 


\section{CONCLUSIONES}

El concepto de Administración del Territorio es poco utilizado a nivel regional, dificultando el adecuado trabajo de los sistemas de información desarrollados para el cumplimiento de sus funciones tradicionales como son la tenencia, la valoración, el uso y desarrollo del territorio; y aún más, la explotación de su verdadero potencial que puede ser aplicado en otras áreas que utilizan como base la información territorial.

La gestión de riesgos en el Ecuador está principalmente enfocada a la respuesta de emergencias y no tanto a la prevención, aun cuando este último ha demostrado ser el mejor y más efectivo método para reducir los efectos devastadores de los desastres naturales, especialmente a través de una óptima planificación y desarrollo del territorio. La falta de una gestión de riesgos integral ha desembocado en la falta de procesos y metodologías capaces de garantizar una adecuada gestión de riesgos, especialmente desde el ámbito territorial.

Existe una estrecha relación entre la administración del territorio y la gestión de riesgos, que se evidenció durante el desarrollo del presente trabajo, al mostrar la utilidad y aplicabilidad de la información y herramientas utilizadas, como el modelo LADM, los sistemas catastrales, elementos informáticos como el STDM y la capacidad de generar normas y políticas territoriales. El uso de información y aplicación de herramientas utilizadas por los sistemas de administración territorial en el proceso de gestión de riesgos permite una planificación territorial más eficiente al considerar acciones que ayuden a reducir el riesgo por peligros de origen natural, así como la difusión de la información disponible a todos los interesados que requieren de ella.

El modelo LADM permite trabajar de acuerdo con diferentes niveles territoriales, de los que dependen los derechos, responsabilidades y restricciones que pueden ser tomados. Para el caso de estudio presentado, el nivel óptimo fue el de los GADs municipales, ya que al ser uno de los objetivos alcanzar una gestión de riesgos a nivel predial. Por ley, los GADs municipales en el Ecuador están a cargo de los catastros inmobiliarios, además de la gestión de riesgos dentro de su circunscripción territorial; cuentan con la información así como con las herramientas necesarias para la aplicación de la metodología propuesta para cumplir de forma óptima con sus obligaciones.

Las herramientas de administración territorial no solo ayudan en el proceso de gestión de riesgos, sino que facilitan la gestión de la información resultante de este procedimiento, permitiendo asentar la planificación propuesta de forma efectiva en el territorio, como se demostró a través de la generación de un sistema catastral basado en el modelo LADM, donde el riesgo determinó nuevos derechos, restricciones y responsabilidades para quienes ejercen la tenencia de los predios. Además, los sistemas catastrales permiten poner en conocimiento de los diferentes usuarios la información referente a riesgos, como se hizo a través del SCR y la generación de Informes Prediales de Riesgo.

La metodología propuesta para la gestión de riesgos naturales en el territorio permitió obtener una completa valoración de los potenciales daños relacionados con el peligro volcánico de los lahares del volcán Cotopaxi en el valle de Los Chillos, evidenciándose que el 100\% de las construcciones de las zonas 1 y 2, ubicadas a las riberas del río Pita se encuentran funcionalmente comprometidas; Rumiñahui es el cantón con mayor afectación y pérdidas económicas. El servicio de agua potable es el más afectado, llegado a ocasionar potenciales racionamientos de líquido vital para el 32,79\% de la población urbana de Quito y de más del $50 \%$ de la población urbana de Rumiñahui, en ambos casos por varios meses. 


\section{REFERENCIAS}

Aguilera, E. y Toulkeridis, T. (2005) Prevención de riesgos asociados con la erupción del volcán cotopaxi 2004 - 2005. Quito: Fundación para el Desarrollo Socioambiental FOES.

Baas, S., Ramasamy, S., Dey de Pryck, J. y Battista, F. (2008) Disaster risk management systems analysis. 13 ed. Roma: FAO Environment and Natural Resources Service Series.

Correa, E., Ramírez, F. y Sanahuja, H. (2011) Populations at risk of disaster: a resettlement guide. Whashington: The World Bank.

Intergovernmental Panel on Climate Change-IPCC. (2014) Informe de evaluación del grupo intergubernamental de expertos sobre el cambio climático. Ginebra: IPCC.

Mitchell, D. (2011) Assessing and responding to land tenure issues in disaster risk management: training manual". Roma: FAO.

Mitchell, D., Myers, M. y Grant, D. (2014) " Land valuation: a key tool for disaster risk management". LAND TENURE JOURNAL, Volumen I, pp. 36-69.

Narváez, C. (2007) Desarrollo de la metodología para el análisis de vulnerabilidad ante una eventual erupción del volcán Cotopaxi. Quito: Escuela Politécnica Nacional, Escuela de Postgrado en Ingeniería y Ciencias.

Organización de Esatdos Americanos - OEA. (1991) Desastres, planificación y desarrollo: manejo de amenazas naturales para reducir los daños. Washington: Organización de los Estados Americanos.

Padilla, O. (2017) Cálculo de los tiempos de evacuación horizontal y vertical en caso de una eventual erupción del volcán Cotopaxi. Alcalá de Henares(Madrid): Universidad de Alcalá; Departamento de Geología, Geografía y Medio Ambiente.

Peña, M. (2016) Análisis de vulnerabilidad y mitigación de riesgos estructurales en las infraestructuras de abastecimiento de agua, en el Distrito Metropolitano de Quito (DMQ), en caso de erupción del volcán Cotopaxi (Ecuador). Madrid: Universidad Politécnica de Madrid, Escuela Técnica Superior de Ingeniería Civil.

Potts, K. E. (2013) Using land administration for land risk management. Melbourne(Victoria): The University of Melbourne.

Rodriguez, F. y otros. (2016) "Economic risk assessment of Cotopaxi volcano, Ecuador, in case of a future lahar emplacement". Natural Hazards, pp. 605-618.

Rojas, R. (2013) Metodología para incorporar cambio climático y gestión del riesgo de desastres en procesos de ot. s.1.:GIZ GmbH-Deutsche Gesellschaft für Internationale Zusammenarbeit.

Saunders, W., Beban, J. y Kilvington, M. (2013) Risk-based land use planning for natural hazard risk reduction. Lower Hutt: Institute of Geological and Nuclear Sciences Limited.

Saunders, W. y Kilvington, M. (2016) "Innovative land use planning for natural hazard risk reduction: a consequence-driven approach from New Zealand". International Journal of Disaster Risk Reduction, Issue 18, pp. 244-255. 
Tibanlombo, J. y Villacís, A. (2013) Aplicación y sistematización de la propuesta metodológica para el análisis de vulnerabilidad de la parroquia Sangolquí, del cantón Rumiñahui. Sangolquí: Universidad de las Fuerzas Armadas - ESPE, Carrera de Ingeniería Geográfica y del Medio Ambiente.

Unger, E. y otros. (2019) Creating resilience to natural disasters through ffp land administration - an application in Nepal. Washington DC: 20th Annual World Bank Conference on Land and Poverty 2019.

Unger, E. M., Zevenbergen, J., Bennett, R. y Lemmen, C. (2019) "Application of LADM for disaster prone areas and communities". Land Use Policy, January, 80, pp. 118-126.

UNISDR AM. (2016) Impacto de los desastres en América Latina y el Caribe, 1990 - 2013. Panamá: United Nations Office for Disaster Risk Reduction - Regional Office for the Americas (UNISDR AM).

Vargas, D. y Ramírez, F. (2009) Incorporando la gestión del riesgo de desastres en la planificación y gestión territorial. Lima: Proyecto Apoyo a la Prevención de Desastres en la Comunidad Andina PREDECAN. 